\title{
A atuação do enfermeiro frente à sexualidade na terceira idade: uma revisão integrativa
}

\author{
The nurse in front of sexuality in old age: an integrative review
}

La acción de enfermería frente a la sexualidad en la tercera edad: una revisión integrativa

Rosane Pereira Dos Reis ${ }^{1 *}$, Josian Karen Claudino Oliveira ${ }^{1}$, Maiza Gomes Vanderlei ${ }^{1}$, Douglas Ferreira Rocha Barbosa ${ }^{1}$, Jirliane Martins Dos Santos ${ }^{1}$, Marcelle Perdigão Gomes ${ }^{1}$, Alexandre De Souza Lima1, Renné Cosmo da Costa ${ }^{2}$, Paulo Jorge torres Guimarães Silva², Daniele Gonçalves Bezerra ${ }^{3}$.

\section{RESUMO}

Objetivo: Descrever a atuação do enfermeiro frente à sexualidade na terceira idade. Métodos: Trata-se de uma revisão integrativa, em que foram consultadas junto à Biblioteca Virtual de Saúde (BVS) acessando as bases de dados: Literatura Latino-Americana e do Caribe em Ciências da Saúde (LILACS), Scientific Eletronic Library Online (SciELO), Cochrane e a Base de Dados em Enfermagem (BDENF), totalizando 16 artigos. Resultados: Os artigos evidenciaram que enfermeiro necessita se conscientizar que a vida sexual do idoso precisa ser vista como realidade para que orientação sobre medidas preventivas das IST's/AIDS possam ser realizadas. Os resultados demonstram ainda que o enfermeiro possui papel fundamental na frente à sexualidade na terceira idade, uma vez que, o mesmo necessita estar habilitado para orientar e abordar este tema com os idosos, visto que faz parte de seus papéis prestarem à assistência humanizada e de qualidade. Considerações finais: Os achados desse estudo permite concluir que a atuação do enfermeiro na assistência sexual dos idosos precisa ser de forma continua, para que as orientações sobre saúde e bemestar dos mesmos, possam ser realizadas, bem como desenvolver atividades de educação em saúde, focando nas medidas preventivas em relação às IST's/AIDS na terceira idade.

Palavras-chave: Idoso, Enfermagem, Sexualidade.

\begin{abstract}
Objective: To describe the role of nurses in relation to sexuality in the elderly. Methods: This is an integrative review, in which they were consulted with the Virtual Health Library (VHL) accessing the databases: Latin American and Caribbean Literature on Health Sciences (LILACS), Scientific Electronic Library Online (SciELO), Cochrane and the Nursing Database (BDENF), totaling 16 articles. Results: The articles showed that nurses need to be aware that the sexual life of the elderly needs to be seen as a reality so that guidance on preventive measures of STIS/AIDS can be performed. The results also show that nurses have a fundamental role in the face of sexuality in the elderly, since they need to be qualified to guide and address this theme with the elderly, since it is part of their roles to provide humanized and quality care. Final considerations: The findings of this study allow us to conclude that the role of nurses in the sexual care of the elderly needs to be continuous, so that the orientations on their health and well-being can be performed, as well as develop health education activities, focusing on preventive measures in relation to STIs/AIDS in the elderly.
\end{abstract}

Key words: Elderly, Nursing, Sexuality.

${ }_{1}^{1}$ Faculdade Estácio de Alagoas (FAL), Maceió - AL. *E-mail: rosane_pr@hotmail.com

${ }^{2}$ Conselho Regional de Enfermagem de Alagoas (COREN-AL), Maceió - AL.

3 Universidade Federal de Alagoas (UFAL), Maceió - AL. 


\section{RESUMEN}

Objetivo: Describir el papel de las enfermeras en relación con la sexualidad en los ancianos. Métodos: Se trata de una revisión integradora, en la que se consultaron con la Biblioteca Virtual de Salud (VHL) accediendo a las bases de datos: Literatura Latinoamericana y del Caribe sobre Ciencias de la Salud (LILACS), Biblioteca Electrónica Científica En Línea (SciELO), Cochrane y la Base de Datos de Enfermería (BDENF), con un total de 16 artículos. Resultados: Los artículos mostraron que las enfermeras deben ser conscientes de que la vida sexual de los ancianos debe ser vista como una realidad para que se puedan realizar orientaciones sobre las medidas preventivas de las INFECCIONes/SIDA. Los resultados también muestran que las enfermeras tienen un papel fundamental frente a la sexualidad en los ancianos, ya que necesitan estar cualificadas para guiar y abordar este tema con los ancianos, ya que es parte de sus funciones para proporcionar una atención humanizada y de calidad. Consideraciones finales: Las conclusiones de este estudio nos permiten concluir que el papel de las enfermeras en la atención sexual de las personas mayores debe ser continuo, de modo que se puedan realizar las orientaciones sobre su salud y bienestar, así como desarrollar actividades de educación sanitaria, centrándose en medidas preventivas en relación con las INFECCIONes/SIDA en los ancianos.

Palabras clave: Ancianos, Enfermería, Sexualidad.

\section{INTRODUÇÃO}

A Organização Mundial da Saúde (OMS), define o idoso como uma pessoa com idade igual a 65 anos ou mais nos países desenvolvidos, já nos países em desenvolvimento, como é o caso do Brasil, a terceira idade é definida quando a pessoa tem mais de 60 anos. Nas últimas décadas tem se observado um expressivo aumento da população idosa. Em 2050, a expectativa de vida da população idosa corresponderá um quarto da população mundial projetada, ou seja, a mais de dois bilhões de pessoas (ORGANIZAÇÃO MUNDIAL DA SAÚDE, 2005).

O Brasil está deixando de ser uma nação de jovens e tornando-se um país com alto contingente de idosos, que cresce em ritmo acelerado (SANTOS AS, et al., 2014). Observa-se a intensificação da proporção de idosos na população brasileira, uma vez que, o envelhecimento é um processo natural que submete o organismo a diferentes mudanças físicas e funcionais. Essas alterações são progressivas e causam efetivas reduções na capacidade funcional do organismo. Embora existam mudanças caracteristicamente relacionadas ao envelhecimento, nem todos os órgãos sofrem seus efeitos da mesma forma; elas acontecem em velocidade diversa e com extensões irregulares (LUZ ACG, et al., 2015).

Para a gerontologia, o envelhecimento pode ser definido como uma sequência da vida, tendo suas particularidades e características. Felizmente, atualmente vem se edificando uma visão mais positiva e bemsucedida para o idoso. Contudo, quando a questão é a sexualidade nesse momento da vida, o tema é cercado de tabus diante da sociedade e até mesmo entre idosos que vivem com mitos e preconceitos. Essa visão desvirtuada é fruto de uma educação muito rigorosa, cheia de julgamentos e preconceitos repressores (LUZ ACG, et al., 2015).

De acordo com Oliveira LB, et al. (2015), a sexualidade quando relacionada ao envelhecimento remete lendas e estereótipos, pois a pessoa idosa é vista pela sociedade como pessoas que não tem interesses sexuais, e consequentemente representando um tabu. Mesmo com a revolução na concepção e na prática da sexualidade ainda podemos registrar preconceito em relação ao atendimento dessas necessidades pelos profissionais de saúde.

Alguns autores trazem entendimento que o preconceito do sexo na velhice é adotado por se acreditar que a fase de vivenciar a sexualidade está condicionada à idade dos mais jovens (ALENCAR DL, et al., 2014). Quando se chega à vida idosa o corpo não responde mais ao desejo tornando-se necessárias adaptações que irá ajudar o idoso na expressão da sexualidade, não limitando a sexualidade somente ao ato sexual, penetração, e se faz necessário a separação de genitalidade e sexualidade. Para muitos, a terceira idade é vista como um período de assexualidade. Alguns idosos manifestam dificuldade em falar sobre sexualidade, assim, esquivam-se do assunto que os deixam constrangidos, revelando o preconceito socialmente construído (SANTOS AS, et al., 2014). 
O enfermeiro por estar inserido neste panorama de saúde é importante que conheça as modalidades dos idosos para contribuir, ajudar e orientá-los, respeitando as singularidades e limitações de cada pessoa (LIMA CFM, et al., 2015).

Dessa forma, ressaltar-se a importância de reconhecer os valores e a cultura dos indivíduos e, desse modo, promover campanhas com direcionamentos diferentes ao público jovem e aos idosos, para se obter resultados mais efetivos em termos de prevenção, de uma atividade sexual segura, promovendo saúde à população de forma mais equânime (SANTOS AS, et al., 2014).

Assuntos pertinentes à saúde sexual da pessoa idosa passam despercebidos durante o atendimento, já que na maioria das vezes, deixa-se de explanar o usuário sobre sexualidade, o que não permite a prevenção de agravos comuns na terceira idade, como a disfunção erétil, o vaginismo, a dispareunia, o uso impróprio de certos remédios e a prevenção da Síndrome da Imunodeficiência Adquirida (AIDS), não concretizando, deste modo, a promoção da saúde dessas pessoas, no sentido de garantir melhor qualidade de vida e comodidade (CUNHA LM, et al., 2015).

Com o aumento da expectativa de vida os idosos estão mais vulneráveis as possíveis consequências das práticas sexuais inseguras, estando exposta a contaminação pelo vírus da imunodeficiência humana (HIV) e outras Infecções Sexualmente Transmissíveis (IST'S) (NETO JD, et al., 2014).

No entanto, as atividades de educação em saúde têm sido estratégias relevantes para a proximidade maior entre profissional e comunidade, possibilitando troca de conhecimentos e retirada de dúvidas (SANTOS AS, et al., 2014).

O interesse pela temática surgiu a partir de convívio com idosos e situações vivenciadas durante o estágio obrigatório em uma Unidade de Saúde da Família (USF) onde foi observado o despreparo profissional da equipe de saúde para lidar com assuntos referentes à sexualidade do idoso, bem como o elevado número de idosos com IST'S, mostrando assim a vulnerabilidade dos idosos diante de práticas sexuais inseguras e a não abordagem de conceitos relacionados à sexualidade durante a consulta de enfermagem.

Portanto, considera-se o estudo de suma importância, visto que possui como foco a sexualidade em toda sua amplitude e traz para o centro das discussões a sexualidade na terceira idade, mostrando como a sexualidade pode vir a impactar diretamente a vida dos idosos e que assim um processo de transformação seja iniciado ainda na vida acadêmica, para que o mesmo possa ser estendido à vida profissional e que 0 reflexo consiga chegar até a realidade social onde a temática sobre sexualidade possa ser incluída durante o atendimento a pessoa idosa, proporcionando um atendimento integral à saúde.

A partir deste contexto e diante do conhecimento produzido sobre o assunto, pretende-se realizar um estudo de revisão integrativa da literatura, levantando a seguinte norteadora: Qual a atuação do enfermeiro frente à sexualidade na terceira idade? Sendo assim, o objetivo desta pesquisa é descrever a atuação do enfermeiro frente à sexualidade na terceira idade.

\section{MÉTODOS}

Trata-se de revisão integrativa da literatura, sendo um método de pesquisa que busca, avalia criticamente e sintetiza a produção sobre determinada temática, de maneira sistemática. Utilizaram-se as seguintes etapas: seleção das questões norteadoras; busca e seleção da literatura nas bases de dados eletrônicas, baseando-se em critérios de inclusão e exclusão da amostra; elaboração de instrumento que inclua as informações relevantes extraídas das amostras; análise crítica dos estudos selecionados; interpretação dos dados; e apresentação dos resultados evidenciados (ERCOLE FF, et al., 2014).

A busca dos estudos foi realizada acessando as seguintes bases de dados Literatura Latino-Americana e do Caribe em Ciências da Saúde (LILACS), Scientific Eletronic Library Online (SciELO) e a Base de Dados em Enfermagem (BDENF) no período de março a abril de 2019. Os critérios de inclusão foram: publicações que estivessem disponíveis na íntegra, gratuito, no período de 2014 a 2018, nos idiomas: português, inglês e espanhol e que respondesse à questão norteadora do estudo. 
Também foram utilizados artigos que não estão na amostra, devido à importância de seu conteúdo para o presente estudo. E os critérios de exclusão foram: cartas ao editor, artigos de opinião, estudos de caso, teses, dissertações, artigos que referenciavam idosos institucionalizados, assim como estudos sem metodologia clara e com impossibilidade de acesso à publicação completa impressa ou on-line e publicações repetidas (entre as bases de dados).

Foi utilizada a seguinte estratégia de busca realizada buscas em bases científicas, utilizando o formulário de busca avançada nas bases de dados, mediante o emprego dos Descritores em Ciências da Saúde (DECS): "Idoso", "Enfermagem", "sexualidade", combinados com o operador booleano "AND" junto aos critérios de inclusão aplicados para composição da amostra. Para interpretação crítica dos artigos, procedeu-se à análise de conteúdo, com discussões entre os autores, obtendo ao final consenso acerca do conteúdo apresentado.

Após a leitura de títulos e resumos, foram selecionados os artigos para leitura na íntegra, tomando como referência a questão do estudo, seguido da interpretação e comparação entre produções e os elementos que compunham cada uma, encontrando informações e evidências relevantes que dissertaram acerca de como a literatura tem referido sobre a atuação do enfermeiro frente à sexualidade na terceira idade.

\section{RESULTADOS}

Na primeira seleção, foram encontrados 2.369 artigos científicos, dos quais 1062 foram no LILACS, 860 no BDENF e 447 no SCIELO. Após avaliação inicial, foram excluídos 2.257 de acordo com o recorte temporal (1014 no LILACS, 823 no BDENF e 423 no SCIELO). Prosseguiu-se, então, para a seleção por títulos de acordo com a temática do estudo e na sequência foi realizada a seleção pela leitura dos resumos, reduzindose a 112 títulos. Também foram identificadas e excluídas 73 duplicadas, 13 títulos não respondiam á questão da pesquisa, 10 títulos eram revisões integrativas de literaturas, chegando ao total de 15 títulos em total como base final (Tabela 1).

Tabela 1 - Dados dos artigos encontrados para revisão de literatura, Maceió, AL, Brasil, 2019.

\begin{tabular}{ccccc}
\hline \multicolumn{5}{c}{ Pareamento dos descritores } \\
LILACS & SCIELO & BDENF & TOTAL \\
\hline Descritores/Fontes & 54 & 16 & 30 & 100 \\
Idoso and sexualidade & 80 & 51 & 76 & 207 \\
Enfermagem and sexualidade & 928 & 380 & 754 & 2062 \\
Enfermagem and idoso & 1062 & 447 & 860 & 2369 \\
\hline Total & & & &
\end{tabular}

Fonte: dos Reis RP, et al., 2019.

Inicialmente foi realizada uma leitura dos resumos dos artigos, escolhendo os de maior pertinência. Depois foi realizada uma segunda leitura para melhor interpretação, dando especial atenção aos resultados e conclusões. Deste modo, prosseguimos para a interpretação dos textos, sendo eles lidos, avaliados e organizados. Em seguida foram destacados os pontos que atendiam à finalidade proposta para a elaboração do texto final.

Quanto às bases de dados, a maior parte dos artigos foram encontrados no BDENF $52,94 \%$ (07 artigos), LILACS 35,29\% (6 artigos) e no SCIELO 11,76\% (2 artigos). Os artigos foram categorizados quanto ao paradigma de área temática, sendo todos relacionados á sexualidade do idoso: 58,82\% (10 artigos) sobre sexualidade do idoso, 23,52\% (3 artigos) sexualidade e assistência de enfermagem ao idoso e 11,76\% (2 artigos) envelhecimento.

$\mathrm{Na}$ presente revisão integrativa quinze artigos atenderam rigorosamente à seleção da amostra previamente estabelecida. A Quadro 1 abaixo apresenta os artigos selecionados em ordem cronológica de publicação. No que concerne às revistas que obtiveram mais publicações foi possível observar uma diversidade de periódicos que publicaram artigos sobre a temática. 
Quadro 1 - Artigos científicos selecionados nas bases de dados LILACS, SCIELO e BDENF segundo o título, periódico e ano. Maceió, 2019.

\begin{tabular}{|c|c|c|c|c|}
\hline $\mathbf{N}$ & Bases de dados & Título do artigo & Periódico/ Ano & Objetivo \\
\hline 1 & \multirow{6}{*}{ LILACS } & $\begin{array}{l}\text { A sexualidade sob o olhar da } \\
\text { pessoa idosa. }\end{array}$ & $\begin{array}{l}\text { Revista brasileira de } \\
\text { geriatria e } \\
\text { gerontologia/2016. }\end{array}$ & $\begin{array}{c}\text { Identificar a percepção dos idosos acerca da } \\
\text { sexualidade. }\end{array}$ \\
\hline 2 & & $\begin{array}{l}\text { O exercício da sexualidade entre os } \\
\text { idosos e fatores associados. }\end{array}$ & $\begin{array}{l}\text { Revista brasileira de } \\
\text { geriatria e } \\
\text { gerontologia/2016. }\end{array}$ & $\begin{array}{l}\text { Analisar os fatores que interferem no exercício da } \\
\text { sexualidade de pessoas idosas. }\end{array}$ \\
\hline 3 & & $\begin{array}{l}\text { Vivência da sexualidade por } \\
\text { mulheres idosas. }\end{array}$ & $\begin{array}{l}\text { Revista brasileira de } \\
\text { geriatria e } \\
\text { gerontologia/2016. }\end{array}$ & $\begin{array}{l}\text { Interpretar a vivência da sexualidade pela mulher } \\
\text { idosa e construir um modelo teórico explicativo. }\end{array}$ \\
\hline 4 & & $\begin{array}{l}\text { O percurso educativo dialógico } \\
\text { como estratégia de cuidado com } \\
\text { mulheres idosas na sexualidade. }\end{array}$ & $\begin{array}{l}\text { Escola Anna } \\
\text { Nery/2018. }\end{array}$ & $\begin{array}{c}\text { Desvelar o conhecimento crítico mediado por um } \\
\text { percurso cuidativo-educativo dialógico em } \\
\text { sexualidade com mulheres idosas. }\end{array}$ \\
\hline 5 & & $\begin{array}{l}\text { A Sexualidade Na Velhice: } \\
\text { Representações Sociais De Idosos } \\
\text { frequentadores de Um Grupo de } \\
\text { Convivência. }\end{array}$ & $\begin{array}{l}\text { Psicologia: ciência e } \\
\text { Profissão /2016. }\end{array}$ & $\begin{array}{c}\text { Apreender as representações sociais dos idosos } \\
\text { acerca da sexualidade. }\end{array}$ \\
\hline 6 & & $\begin{array}{l}\text { El deseo sexual en varones adultos } \\
\text { mayores, su relación con la } \\
\text { testosterona sérica y otros factores. }\end{array}$ & $\begin{array}{l}\text { Revista Cubana de } \\
\text { Endocrinologia/2016. }\end{array}$ & $\begin{array}{c}\text { Describir las características del deseo sexual en } \\
\text { varones adultos mayores del municipio Plaza de la } \\
\text { Revolución y su asociación con la testosterona } \\
\text { sérica y otras variables. }\end{array}$ \\
\hline 7 & \multirow{2}{*}{ SCIELO } & $\begin{array}{c}\text { Avaliação da abordagem médica da } \\
\text { sexualidade em idosos com dor } \\
\text { crônica. }\end{array}$ & Einstein /2016. & $\begin{array}{l}\text { Determinar a frequência com que os médicos } \\
\text { abordam o assunto de sexualidade com seus } \\
\text { pacientes mais idosos com dor crônica. }\end{array}$ \\
\hline 8 & & $\begin{array}{l}\text { A vivência da sexualidade por } \\
\text { idosas viúvas e suas percepções } \\
\text { quanto à opinião dos familiares a } \\
\text { respeito. }\end{array}$ & $\begin{array}{l}\text { Revista Saúde } \\
\text { Sociedade São Paulo } \\
\text { / } 2015 .\end{array}$ & $\begin{array}{l}\text { O objetivo deste trabalho foi descrever a vivência } \\
\text { da sexualidade por mulheres idosas viúvas, } \\
\text { frequentadoras de um Centro de Convivência do } \\
\text { Idoso, e verificar a percepção quanto à opinião dos } \\
\text { seus familiares. }\end{array}$ \\
\hline
\end{tabular}

REAS/EJCH | Vol.Sup.n.55 | e3740 | DOI: https://doi.org/10.25248/reas.e3740.2020 Página 5 de 10 


\begin{tabular}{|c|c|c|c|c|}
\hline $\mathbf{N}$ & Bases de dados & Título do artigo & Periódico/ Ano & Objetivo \\
\hline 9 & \multirow{7}{*}{ BDENF } & $\begin{array}{c}\text { Cuidado terapêutico de } \\
\text { enfermagem: Transições da } \\
\text { sexualidade do cônjuge-cuidador do } \\
\text { idoso. }\end{array}$ & $\begin{array}{l}\text { Revista Brasileira. } \\
\text { Enfermagem/2017. }\end{array}$ & $\begin{array}{l}\text { Compreender as transições vivenciadas, suas } \\
\text { condições e os padrões de resposta esperados a } \\
\text { mudanças na sexualidade do cônjuge-cuidador do } \\
\text { idoso em processo demencial. }\end{array}$ \\
\hline 10 & & $\begin{array}{l}\text { Representações sociais da } \\
\text { sexualidade entre idosos. }\end{array}$ & $\begin{array}{l}\text { Revista Brasileira } \\
\text { Enfermagem/2015. }\end{array}$ & $\begin{array}{c}\text { Conhecer a representação social sobre } \\
\text { sexualidade de idosos, com base na Teoria das } \\
\text { Representações Sociais. }\end{array}$ \\
\hline 11 & & $\begin{array}{l}\text { Sexuality and aging: identified } \\
\text { needs for construction of an } \\
\text { educational technology. }\end{array}$ & $\begin{array}{l}\text { Journal of Nursing } \\
\text { UFPE on line/2017 }\end{array}$ & $\begin{array}{c}\text { To identify the needs of the elderly in view of their } \\
\text { sexuality to subsidize the construction of an } \\
\text { educational technology. }\end{array}$ \\
\hline 12 & & $\begin{array}{l}\text { A vivência da sexualidade de idosos } \\
\text { em um centro de convivência. }\end{array}$ & $\begin{array}{l}\text { Revista Enfermagem } \\
\text { centro oeste } \\
\text { mineiro/2015. }\end{array}$ & $\begin{array}{l}\text { Conhecer como o idoso desse Centro de } \\
\text { Convivência vive sua sexualidade e discutir a sua } \\
\text { percepção quanto à prática sexual e compreender } \\
\text { como ele vivencia esse momento. }\end{array}$ \\
\hline 13 & & $\begin{array}{l}\text { Sexualidade na terceira idade: } \\
\text { percepção de homens idosos de } \\
\text { uma estratégia de saúde da família. }\end{array}$ & $\begin{array}{l}\text { Journal of Nursing and } \\
\text { Health/2015. }\end{array}$ & $\begin{array}{l}\text { Identificar o conhecimento e os fatores que } \\
\text { interferem na sexualidade de homens idosos. }\end{array}$ \\
\hline 14 & & $\begin{array}{l}\text { Vovó e vovô também amam: } \\
\text { sexualidade na terceira idade }\end{array}$ & $\begin{array}{l}\text { Revista Mineira de } \\
\text { Enfermagem/2015. }\end{array}$ & $\begin{array}{c}\text { Objetivou-se analisar a prática profissional de } \\
\text { médicos e enfermeiros da Estratégia Saúde da } \\
\text { Família no que se refere aos aspectos da } \\
\text { sexualidade em idosos. }\end{array}$ \\
\hline 15 & & $\begin{array}{l}\text { Comportamento sexual de idosos } \\
\text { assistidos na estratégia saúde da } \\
\text { família. }\end{array}$ & $\begin{array}{l}\text { Revista de Pesquisa } \\
\text { Cuidado é } \\
\text { Fundamental } \\
\text { Online/2015. }\end{array}$ & $\begin{array}{l}\text { Analisar o comportamento sexual de idosos } \\
\text { assistidos na atenção primária em saúde. }\end{array}$ \\
\hline
\end{tabular}

Fonte: dos Reis RP, et al., 2019. 


\section{DISCUSSÃO}

Dentre as definições encontradas, entende-se que nos próximos vintes anos a população com mais de 60 anos triplicará com o provável aumento no tempo de vida, sendo assim necessário um adequado acompanhamento no processo de envelhecimento levando em consideração fatores que poderão interferir na qualidade de vida da pessoa na terceira idade, compreendendo também o envelhecer como um processo natural e possível de uma assistência integral a saúde (UCHÔA YS, et al., 2016).

Segundo Marques ADB, et al. (2015) o indivíduo precisa perceber seu contexto cultural e os valores da sociedade em que vive, para assim possa desenvolver seus objetivos, expectativas, padrões e preocupações sobre a qualidade de vida e dentro desse conhecimento também está inserido a sexualidade.

Conforme Luz ACG, et al. (2015) a sociedade ocidental vê o envelhecimento como sinônimo de incapacidade, de doenças e uma possível morte, e dentro dessa visão negativa também está à sexualidade na terceira idade. Deste modo, Nascimento RF, et al. (2017) afirma que a personalidade do indivíduo é estruturada pela sexualidade é através da sexualidade que o indivíduo externa seu sexo.

A sexualidade é uma ação que tem ligação direta com uma necessidade fisiológica, não se resume apenas ao ato sexual. Ainda nesta mesma linha de considerações Araújo MIR, et al. (2017), afirma que dentre os direitos humanos universais está inserida a sexualidade, e que direitos sexuais são fundamentais para 0 desenvolvimento do bem-estar individual e interpessoal.

De acordo com Souza MD, et al. (2015) pelo fato de a sexualidade não ser um tema discutido durante a sua juventude, os idosos sentem-se desconfortáveis quando se fala sobre o assunto mesmo sendo indispensável abordar sobre sexualidade em qualquer etapa da vida.

A sexualidade quando relacionada ao envelhecimento manifesta mitos e preconceitos, derivando na percepção de que os idosos são pessoas assexuadas. Diante disso, a sexualidade do idoso precisa ser compreendida deste sujeito, necessitando o idoso ser analisado como um todo, ou seja, no sentido holístico (QUEIROZ MAC, et al., 2015). A vivência da sexualidade na terceira idade se reflete sempre na amplitude e na facilidade com que se entregam, pois eles acabam aceitando a se completar e a serem completados.

Os idosos não recebem a devida atenção da sociedade e dos profissionais da saúde quando o assunto é sexualidade na terceira idade, cria-se uma imagem preconceituosa revelando um receio em abordar a temática, o tema sexualidade é de suma importância para abordar e assim serem encontrados e discutidos possíveis ou já existentes alterações relacionadas à sexualidade (LUZ ACG, et al., 2015). É importante também que o enfermeiro se aprimore para abordar temas de sexualidade com os pacientes idosos, permitindo um ambiente em que eles sintam confiança, possam adquirir informações e tirar dúvidas para que passem por essa etapa com qualidade de vida sexual.

As alterações fisiológicas são esperadas na terceira idade, devido ao processo natural do envelhecimento que podem exercer influência na resposta sexual, sejam do sexo masculino ou feminino. As mudanças na fisiologia sexual masculina embora não aconteçam de forma monótona entre todos os homens caracterizamse quanto aos seguintes aspectos: ereção mais flácida, sendo necessário mais tempo para alcançar o orgasmo; redução das ereções involuntárias noturnas; ejaculação demorada e diminuição do líquido préejaculatório.

Já nas mulheres o envelhecimento fisiológico produz as seguintes mudanças: estreitamento das paredes vaginais, redução da elasticidade da vagina, diminuição da lubrificação vaginal, diminuições das contrações vaginais e uterinas durante orgasmo (CHERPARK GL e SANTOS FCD, 2016). Diante dessas mudanças, o enfermeiro precisa estar habilitado para reconhecer estas alterações que ocorrem devido ao processo de envelhecimento para instituir e compreender os questionamentos apresentados pelos idosos a fim de proporcionar uma assistência holística.

O estudo realizado por Rodrigues DMMR, et al. (2018), faz referência que à proporção que as mulheres envelhecem e perdem seus níveis de esteroides sexuais, as contrações uterinas que acontecem com o orgasmo se tornam frequentemente doloridas, e esta cólica uterina se desenvolve tanto durante a expressão orgásmica quanto após a mesma. 
Os fatores psicológicos estão ligados a não aceitar da terceira idade, que de certa forma, representa algo natural, mas influência negativamente na qualidade de vida dessa população (PEÑA GM, et al., 2016). Conforme o autor, quando o ver-se e o sentir-se idoso não encontram um ponto de interseção, provavelmente não se terá como atribuir estado de velhice à pessoa.

Já as mudanças sociais são marcadas pelo isolamento social, problemas econômicos, como também os diversos outros tipos de limitações com o surgimento das enfermidades crônico-degenerativas e a ideia da senectude e a proximidade iminente da morte comprometem e influenciam negativamente o imaginário em relação à velhice (VIEIRA KFL, et al., 2016).

É importante ressaltar que na maioria das vezes a sexualidade nessa etapa da vida não é levada a sério nos serviços de atenção à saúde do idoso (PEIXER TC, et al., 2015). Deste modo, os profissionais de saúde, em especial os enfermeiros, necessitam reconhecer que as alterações são decorrentes do processo de envelhecimento e que isso não extingue a sexualidade, sendo necessário que essas alterações sejam debatidas nas consultas de enfermagem, proporcionando uma melhor assistência ao idoso.

Burigo GF, et al. (2015) relata que devido os progressos da tecnologia e da atenção à saúde, os idosos vivem um fato nunca antes sentido nesse momento da vida. As drogas que atuam no comportamento sexual e as inovações na área da reposição hormonal aumentaram a qualidade e a assiduidade das relações sexuais. Alencar DLD, et al. (2016) comenta que diante dessas mudanças, o enfermeiro precisa estar habilitado para reconhecer estas alterações que ocorrem devido ao processo de envelhecimento para instituir e compreender os questionamentos apresentados pelos idosos a fim de proporcionar uma assistência holística.

Rodrigues DMMR, et al. (2018) salienta que o enfermeiro é de grande importância no momento da educação em saúde da construção de hábito a uma prática sexual saudável, construir um diálogo entre paciente e profissional a proporcionar um conforto no atendimento com o objetivo de encontrar maneiras que o idoso possa praticar sua sexualidade adequando-se a suas necessidades.

Para Cherpark GL e Santos FCD (2016) a dificuldade dos enfermeiros em falar sobre a sexualidade na terceira idade é evidente, pois o conhecimento e o comportamento em relação às IST's/AIDS são, em geral, discutidos apenas para alguns grupos característicos que eliminam os idosos.

Além disso, a ausência de interesse da equipe de saúde em relação à sexualidade na terceira idade gera dificuldade para abordar e explicar aos idosos sobre tal temática. Essa falha ocasiona graves consequências, especialmente em relação à prevenção, visto que esta só vai acontecer quando os familiares de idosos e profissionais de saúde estiverem atentos para discutir espontaneamente as formas de prevenção (MARQUES ADB, et al., 2015). É necessário que o enfermeiro se conscientize que a vida sexual do idoso precisa ser vista como realidade para que orientação sobre medidas preventivas das IST's possam ser realizadas.

É preciso que as ações de enfermagem como: desenvolver protocolos de atuação e programas de educação a saúde; focalizar medidas preventivas quanto às disfunções eréteis, menopausa, andropausa, divulgar a existência de métodos clínicos e ou cirúrgicos para a expressão da sexualidade, trabalhar diretamente com o idoso despertando o interesse, dando destaque aos seguintes aspectos: atividade sexual, preconceitos, receio, vergonha, culpa e falsas ideologias, sejam adotadas para melhoria da assistência prestada e consequentemente da qualidade de vida desta clientela (CUNHA LM, et al., 2015). Infelizmente a sexualidade na terceira idade ainda é pouco discutida e na maioria das vezes, ignorada.

O enfermeiro precisa realizar um plano de cuidado direcionado a esta população com intervenções como: criar novas estratégias para estimular o interesse e criatividade em relação à sexualidade na terceira idade; buscar táticas para motivar os idosos a descobrirem novas maneiras de satisfação; ouvir atentamente o idoso; deixar o idoso expressar seus sentimentos e angústias (VENTURINI L, et al., 2018). 
Cabe destacar ainda que na maioria das vezes a sexualidade nessa etapa da vida não é levada a sério nos serviços de atenção à saúde do idoso. Deste modo, os profissionais de saúde, em especial os enfermeiros, necessitam reconhecer que as alterações são decorrentes do processo de envelhecimento e que isso não extingue a sexualidade, sendo necessário que essas alterações sejam debatidas nas consultas de enfermagem, proporcionando uma melhor assistência ao idoso.

Portanto, a educação em saúde necessita ser realizada de forma eficaz, para que as táticas utilizadas na constituição de considerações considerem o idoso como indivíduo livre para experienciar sua sexualidade, desprendida de mitos e preconceitos que se consolidaram socialmente. Faz-se necessário fundamentar que o enfermeiro possui papel fundamental na frente à sexualidade na terceira idade, uma vez que, o mesmo necessita estar habilitado para orientar e abordar este tema com os idosos, visto que faz parte de seus papéis prestarem à assistência humanizada e de qualidade.

\section{CONSIDERAÇÕES FINAIS}

Os achados desse estudo permite concluir que a atuação do enfermeiro na assistência sexual dos idosos precisa ser de forma continua, para que as orientações sobre saúde e bem-estar dos mesmos, possam ser realizadas, bem como desenvolver atividades de educação em saúde, focando nas medidas preventivas em relação às IST's/AIDS na terceira idade. Observou-se também que o presente estudo abordou as evidências cientificas que trazem a atuação do enfermeiro frente à sexualidade na terceira idade levando em consideração os aspectos sociais e fatores que podem influenciar na sexualidade do idoso onde foi constatado que o tema é de grande relevância e indica a necessidade de novas pesquisas, que contribuam para uma abordagem cientificamente mais adequada a população idosa quando o assunto é sexualidade e que esse tema seja incluido no cuidado terapêutico de Enfermagem ao idoso e assim eles possam envelhecer de forma natural e saudável.

\section{REFERÊNCIAS}

1. ALENCAR DLD, et al. Fatores que interferem na sexualidade de idosos: uma revisão integrativa. Ciência \& Saúde Coletiva, 2014; 19(8): 3533-3542.

2. ALENCAR DLD, et al. O exercício da sexualidade entre os idosos e fatores associados. Revista Brasileira de Geriatria e Gerontologia, 2016; 19(5): 861-869.

3. ARAUJO MIR, et al. Sexuality and aging: identified needs for cosntruction of na educational technology. Journal of Nursing UFPE/Revista de Enfermagem UFPE, 2017; 11(7).

4. BURIGO GF, et al. Sexualidade e comportamento de idosos vulneráveis a doença sexualmente transmissíveis. RevCuidArte Enfermagem, 2015; 9(2): 148-153.

5. CHERPAK GL, SANTOS FCD. Avaliação da abordagem da sexualidade em idosos com dor crônica. Einstein (São Paulo), 2016; 14(2): 178-184.

6. CUNHA LM, et al. Vovó e vovô também amam: sexualidade na terceira idade. Revista Mineira de Enfermagem, 2015; 19(4): 894-906.

7. ERCOLE FF, et al. Revisão integrativa versus revisão sistemática. Revista Mineira de Enfermagem, 2014; 18(1): 912.

8. LIMA CFM, et al. Sexualidade do cônjuge que cuida do idoso demenciado: revisão integrativa da literatura. Revista Mineira de Enfermagem, 2015; 19(2): 211-224.

9. LIMA CFM, et al. Therapeutic nursing care: transition in sexuality of the elderly caregiving spouse. Revista Mineira de Enfermagem, 2017; 70(4): 673-681.

10. LUZ ACG, et al. Comportamento sexual de idosos assistidos na estratégia saúde da família. Revista de Pesquisa Cuidado é Fundamental Online, 2015; 7(2): 2229-2240.

11. MARQUES ADB, et al. A vivência da sexualidade de idosos em um centro de convivência. Revista de Enfermagem do Centro-Oeste Mineiro, 2015; 5(3): 1768-1783.

12. NASCIMENTO RF, et al. Vivência da sexualidade por mulheres idosas [Sexuality as experiencedbyolderwomen] [Experiencia de lasexualidad por señorasmayores]. Revista Enfermagem UERJ, 2017; 25(e20892): 1-5.

13. NETO JD, et al. Doenças sexualmente transmissíveis em idosos: uma revisão sistemática. Ciência \& Saúde Coletiva, 2015; 20(12): 3853-3864. 
14. OLIVEIRA LB, et al. Sexualidade e envelhecimento: avaliação do perfil sexual de idosos não institucionalizados. Rev. Ciênc. Saúde Nova Esperança, 2015; 13(2): 42-50.

15. ORGANIZAÇÃO MUNDIAL DA SAÚDE. Envelhecimento ativo: uma política de saúde. Organização Pan-Americana da Saúde-OPAS, 2005.

16. PEIXER TC, et al. Sexualidade na terceira idade: percepção de homens idosos de uma estratégia de saúde da família. Sexuality at the third age: perception of elderly men from a family health strategy. J Nurs Health, 2015; 5(2): $131-140$.

17. PEÑA GM, et al. El deseo sexual envarones adultos mayores, surelaciónconla testosterona sérica y otrosfactores. Rev Cubana Endocrinol, Ciudad de la Habana, 2016; 27(1).

18. QUEIROZ MAC, et al. Representações sociais da sexualidade entre idosos. RevBrasEnferm, 2015; 68(4): 662-667.

19. RODRIGUES DMMR, et al. O percurso educativo dialógico como estratégia de cuidado com mulheres idosas na sexualidade. Esc. Anna Nery, 2018; 22(3): 1-7.

20. SANTOS AS, et al. Compreensão de idosos e familiares sobre sexualidade e HIV/Aids: estudo descritivo. OBJNOnline Brazilian Journal of Nursin, 2014; 13(2): 175-185.

21. SOUZA MD, et al. A vivência da sexualidade por idosas viúvas e suas percepções quanto à opinião dos familiares a respeito. Saúde Soc. São Paulo, 2015; 24(3): 936-944.

22. UCHOA YS, et al. A sexualidade sob o olhar da pessoa idosa. Revista Brasileira de Geriatria e Gerontologia, 2016; 19(6): 939-949.

23. VENTURINI L, et al. Atuação da equipe de enfermagem frente à sexualidade de idosas institucionalizadas. Revista da Escola de Enfermagem da USP, 2018; 52: 1-8.

24. VIEIRA KFL, et al. A sexualidade na velhice: representações sociais de idosos frequentadores de um grupo de convivência. Psicologia Ciência e Profissão, 2016; 36(1): 196-209. 